\title{
Self-service Data Science for Healthcare Professionals: A Data Preparation Approach
}

\author{
Marco Spruit ${ }^{1} \mathbb{D}^{\mathrm{a}}$, Thomas Dedding ${ }^{1}$ and Daniel Vijlbrief ${ }^{2}$ \\ ${ }^{I}$ Department of Information and Computing Sciences, Utrecht University, The Netherlands \\ ${ }^{2}$ Department of Neonatology, Wilhelmina Children's Hospital, University Medical Center Utrecht, The Netherlands
}

Keywords: Applied Data Science, Meta-algorithmic Modelling, Knowledge Discovery, Domain Expertise, Healthcare, Data Analytics, CRISP-DM.

\begin{abstract}
Knowledge Discovery and Data Mining are two well-known and still growing fields that, with the advancements of data collection and storage technologies, emerged and expanded with great strength by the many possibilities and benefits that exploring and analyzing data can bring. However, it is a task that requires great domain expertise to really achieve its full potential. Furthermore, it is an activity which is done mainly by data experts who know little about specific domains, like the healthcare sector, for example. Thus, in this research, we propose means for allowing domain experts from the medical domain (e.g. doctors and nurses) to also be actively part of the Knowledge Discovery process, focusing in the Data Preparation phase, and use the specific domain knowledge that they have in order to start unveiling useful information from the data. Hence, a guideline based on the CRISP-DM framework, in the format of methods fragments is proposed to guide these professionals through the KD process.
\end{abstract}

\section{INTRODUCTION}

Knowledge Discovery and Data Mining are two wellknown and steadily expanding fields that investigate the many possibilities and benefits that exploring and analysing data can bring. Knowledge Discovery (KD) was defined by Fayyad, Piatetsky-shapiro, \& Smyth (1996) as "the non-trivial process of identifying valid, novel, potentially useful and ultimately understandable patterns in data". Additionally, Data Mining (DM) was defined by Luo (2008) as "the process of analysing data from different perspectives and summarizing it into useful information". Hence, although sharing similar goals (turning data into knowledge), assuming that KD and DM are the same is a misconception. $\mathrm{KD}$ is an overall process of extracting information from data which can be turned into valuable insights, having the application of DM techniques within it. DM on the other hand, can be addressed as the application of methods, techniques and specific algorithms to extract those useful patterns out of the data. However, extracting knowledge from data is not a trivial task and the process is composed of many phases and activities.
These vary in complexity and importance for each specific and distinct scenario, dataset, and problem. For larger and massive datasets, one of the most problematic and exhaustive tasks is to prepare the data, by removing inconsistences, integrating tables, and transforming its variables and values, before applying the different statistical methods and techniques to obtain useful information from it. This requires a good understanding of the business goals and project's objectives, and also a good understanding of the data itself. Moreover, since data preparation is an activity as important as data mining, lacking in doing it properly can hinder or even compromise the entire data analysis activity. Hence, even for simple questions and hypothesis, preparing the data properly is essential for a good analysis, and to avoid getting bias for not considering 'dirty' data into it.

Although in healthcare research KD's application is currently not as advanced as in other areas, it is already being used to aid nurses and doctors on their daily activities and patient's treatment, where some of the potential benefits of using data smartly and effectively includes detecting and preventing

a(D) https://orcid.org/0000-0002-9237-221X 
potential diseases, predicting outcomes, and estimating length of hospital stay (Raghupathi \& Raghupathi, 2014). For that purpose, hospitals usually seek data experts to help them on extracting knowledge from their data, however, these professionals often do not have the specific medical background information to make the most of their analysis, like doctors would if the right tools were provided to them. Therefore, the data exploration process could take longer than needed due to the fact that the person would not have the proficiency, for example, to fully understand all the variables within the data. Thus, if domain experts could also have a way and be supported to analyse the data, the knowledge discovery process could be greatly improved, as they are supposed to have a deeper understanding of the business, problems, and the attributes within the data. Thus, given the power that data analytics has, and although data scientists and analysts are very much required in today's market, KD should not be an exclusive activity for those professionals, especially because (as stated above) they usually lack relevant domain knowledge when compared to field experts. Hence, based on the above, the overarching research question for this research is:

How can the data preparation phase, embedded within the knowledge discovery process, be further specified to enable domain experts such as healthcare professionals to explore analytical problems more easily and intuitively by themselves?

As defined by Spruit \& Jagesar (2016), Applied Data Science is "the knowledge discovery process in which analytical applications are designed and evaluated to improve the daily practices of domain experts". Therefore, to help domain experts to analyse, understand and extract knowledge from data, and by this, improve their daily practices, we present, in the format of method fragments, a guideline which suggests how domain experts should pursue the data pre-processing phase of the KD process. In addition to that, in order to gather enough information and knowledge over what was important and should be included in the guideline, an extensive literature study was made, together with information collected by means of interviews with domain experts from the medical domain, and a brief data quality assessment over the information collected and stored within the databases from the children's hospital in the city of Utrecht in the Netherlands (WKZ), as will be briefly explained next. The guideline development was based on the CRISP-DM framework, which was adapted to the domain experts' needs. Hence, first, a brief theoretical explanation that supports $\mathrm{KD}$ to be done by domain experts will be given, together with the summarized findings from the interviews and data quality assessment. Next, the CRISP-DM's adaptation will be shown, followed by the developed guideline. Finally, we will present the evaluation results for this study, as well as or conclusions and future steps.

\section{DOMAIN EXPERTS AND KNOWLEDGE DISCOVERY}

As could be seen already, Knowledge Discovery is a complex and extensive process where DM is only one step within it. Even so, data driven activities keep on focusing specially in DM, while the other phases are underestimated and their importance is not really taken seriously (Tsai, Lai, Chao, \& Vasilakos, 2015). That creates a deficiency in what is expected from the business and what is actually delivered. For example: data scientists and researches identify achievements and findings from a technical perspective, while business analysts need useful information that actually add some value to the business. Moreover, organizations then seek to perform Actionable Knowledge Discovery (AKD) instead of simple KD, or in other words, extract knowledge from data that actually supports decision-making and action-taking activities. As per Cao (2012), one of the main concepts (among others) which create the basis for applying AKD is 'ubiquitous intelligence' which makes reference to all knowledge and information surrounding the AKD process. Thus, it can be categorized by in-depth data intelligence, which refers not only to the task of extracting patterns from transactional or demographic data, but the power of adding into the analysis real-time data, multidimensional data, business performance data, environmental data, etc.; domain intelligence, which involves extracting all relevant knowledge from the project's domain, such as expert knowledge, background information, possible constraints, etc.; organizational and social intelligence, which refers to all organizational and social information that can be extracted to and added into the analysis, such as business processes and rules, organograms, etc.; network and web intelligence, which refers to hidden information throughout, for instance, distributed systems, network structures, online communities, emails, etc.; and human intelligence, which refers to the participation of domain experts into the knowledge discovery process, by means of supervising, evaluating, sharing knowledge, and sharing expectations and priorities. 


\subsection{What Domain Experts Know about Knowledge Discovery}

Although is explicit that domain experts are required in order to perform AKD, it was not clear how much these professionals could indeed contribute to the process, or in other words, how much they knew about KD. Hence, as said above, seven semi-structure interviews were conducted in order to gather information about how much medical experts knew about this topic, where the number of participants was chosen following the 'data saturation' theory (Francis et al., 2010). All participants were medical experts with no deep technical expertise, and the interviews covered the following topics:

- Knowledge Discovery Understanding: what the interviewee understands about knowledge discovery, their thoughts about its benefits for the organization, patients, etc., and what is the understanding about the process of discovering knowledge from data;

- Data Preparation and Modeling Understanding: this aimed to understand if the interviewee have any technical knowledge such as statistical and programming skills, their experience in extracting knowledge out of data, their difficulties, and knowledge over the available data;

- Expectations and Thoughts over KD: aiming to understand their expectations of being able to analyze data themselves, if they would be able to do it in their daily work, and their experiences (if any) with third-party data analysts doing data analysis.

As a result, even though the exact definition of $\mathrm{KD}$ was not known, the idea of using data to ex-tract information that can be used to better treat patients, and even prevent diseases to happen was well acknowledged by all the interviewees. Additionally, it was possible to see that some of the concepts and phases from CRISP-DM, for example, such as business understanding, data understanding, data preparation, and modelling could be seen in the answers given by the participants, and therefore most of them had an overall understanding of the activities that exist in between defining a goal and analysing data. Furthermore, as expected, domain experts lack programming skills, and therefore, any analysis based on coding activities can be difficult for them. However, most of them said to have a reasonable knowledge of statistical methods, which on the other hand, allows them to, by using other techniques, analyse data. Hence, their experience in the matter is mostly based on basic exploratory analyses or applying statistical methods for testing research hypothesis. When asked about the quality of the data that they usually work with, most of them complained about the huge amounts of dirty data present in the many databases (given bad inputs from machines or even doctors and nurses who add information e.g. free text, into the databases). Also, most participants had an idea of what data was being stored into the databases but had no clue on how to check that information, or to retrieve such content. However, one of the main difficulties stated by them was to gather information from different places (as the data is scattered throughout many tables) in one single master-file where they could base their analysis upon.

Furthermore, most of them demonstrated excitement when asked if they would like to spend more time analysing data if that activity was somehow facilitated. A recent study made by analysts from Gartner Inc. (Linden et al., 2017) evaluated top-rated commercial data science platforms (i.e. software applications that can produce all types of data science solutions), and showed that almost none of them could support less technical people. When asked about their thoughts and wishes, most domain experts complained about the so called 'black-box' scenario, or not having control (or not completely understanding) about what was being done beneath algorithms within tools and wish to have some stepby-step guidance on how to pursue an analytical task. Moreover, domain experts know the challenges and difficulties of dealing with data and know how essential data experts are for the process. However, they know that most of the times data ana-lysts and scientists don't have the medical background to understand and extract all relevant information from the data, and that therefore, they have to work together with these people, providing them medical knowledge in order to get the "right" answers.

\subsection{Data Quality Understanding}

Much has been said about the benefits of using data analytics as a decision-support mechanism in different areas of application, especially in the healthcare. However, these benefits are directly related to the quality of data that is being used during the analysis, and therefore, people have to be really sure that the data is trustworthy. Nowadays most data within hospitals is being generated by means of electronic health records (EHRs), which should be, most of the times, reliable. However, even those mechanisms sometimes depend on human factors, such as an electrode being connected correctly to a patient. Besides that, a lot of data from, for example, 
the intensive care units, as per the interviewees, are still being entered by doctors and nurses as free-text based on observations or comments that can differ for each professional. Thus, to understand what domain experts would be dealing with when analysing data, and based on Batini, Cappiello, Francalanci, \& Maurino, (2009), four main quality dimensions (which although do not represent all dimensions, are considered the centre of attention for the majority of researchers) were briefly analysed: accuracy, completeness, consistency, and timeliness. The first one refers to syntactic and semantic accuracy for example, which sees if a value is syntactically correct, and if that same value is making reference to what it was supposed to respectively; completeness makes reference to the amount and impact of missing values within a dataset; consistency is when values, attributes, and constraints are persisted across the whole database; and lastly, timeliness refers to how current the data is, and whether the it is available when expected and needed to be.

Table 1: Quality Issues Overview.

\begin{tabular}{|c|c|}
\hline $\begin{array}{l}\text { Quality } \\
\text { Dimensions }\end{array}$ & Type of Problems Found \\
\hline 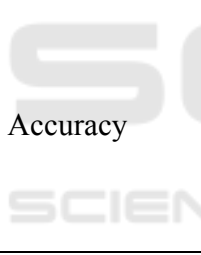 & $\begin{array}{l}\text { Medication being written in up to } \\
\text { seventy different manners. } \\
\text { NICU patients from the gaining up to } \\
\text { three kilograms in the same day. } \\
\text { Length of stay in the NICU of less } \\
\text { than } 5 \text { minutes for some of the } \\
\text { patients. }\end{array}$ \\
\hline Consistency & $\begin{array}{l}\text { Around } 2 \% \text { of the random sample of } \\
16.000 \text { records was duplicated. } \\
\text { Almost } 20 \% \text { of the } 10.647 \text { patients } \\
\text { analysed were officially admitted } \\
\text { after or discharged before a } \\
\text { measurement was realized. } \\
\text { Different units of measurement (e.g. } \\
\text { millilitres, grams, kilograms, etc.) are } \\
\text { being utilized, where for some } \\
\text { specific types of measurements the } \\
\text { unit employed is being specified in } \\
\text { the its name (i.e. grams for measuring } \\
\text { the patient's weight), however, for } \\
\text { others it is very hard to identify it. }\end{array}$ \\
\hline Completeness & $\begin{array}{l}\text { Almost } 24 \% \text { (from a random sample } \\
\text { of } 50.000 \text { records) of the information } \\
\text { about line's type and position was } \\
\text { missing from the data table. } \\
\text { From a random sample of } 12.000 \\
\text { records referring to antibiotic } \\
\text { treatment, the information of which } \\
\text { type of medication was given is } \\
\text { missing in around } 67 \% \text { of the cases. }\end{array}$ \\
\hline Timeliness & $\begin{array}{l}\text { No specific problem was found in this } \\
\text { dimension. }\end{array}$ \\
\hline
\end{tabular}

The data was analysed using $\mathrm{R}$, where by means of an exploratory analysis some problems were discovered. The analysis was made upon the data corresponding to the Ne-onatal Intensive Care Unit (NICU) from the WKZ, without following any specific procedure. Hence, Table 1 summarizes the problems that were found, per quality dimension.

As can be seen, many data problems related to the mentioned quality dimensions were found (due to page restrictions they cannot be exemplified in detail), depicting flaws that probably ex-tend to both data generation process and technical aspects (e.g. better definition of integrity con-strains to avoid human errors). Regarding some examples given above, such as the wrong values concerning the weight of the babies, is hard not to ask further questions such as whether those values were typographical errors, or if, those values belonged to some other patient and were exchanged by mistake; if yes, whether that could be happening to other variables as well and how often. Thus, although many data quality problems exist and can be easily seen, some of them open new questions about the whole validity of the available data, which would require a more extensive data quality assessment to be checked. Nevertheless, with the knowledge that was acquired, it is clear that this matter requires more attention and continuously improvement to slowly transforming and creating a more trustworthy and consistent data environment.

\section{CRISP-DM FRAMEWORK ADAPTATION}

As per the CRISP-DM framework, all main phases of the KD process and their respective out-comes are very well defined (Chapman et al., 2000). However, there is no distinction of how phases should be pursued (and what outcomes are expected) depending of the type of user who is fol-lowing the guideline. For example, the majority of data driven tasks are mainly being done by data analysts and scientists, who spend hours, days, and even weeks, understanding and map-ping inconsistencies and potential problems, and applying DM methods on the data. On the other hand, domain experts usually have different priorities where unfortunately the focus is not KD. Hence, they do not have the same amount of time to spend on data analysis, and therefore, not all $\mathrm{KD}$ phases will be conducted with the same level of details when compared with data ex-perts. Thus, as the focus and the technical knowledge is different, the 
way of pursuing the phas-es from CRISP-DM should be different as well.

Additionally, in order to facilitate the Data Preparation phase, the Data Understanding phase has to be considered as well. As mentioned in the last section, the main aspect that differentiates domain experts from the majority of external analysts, is the huge domain knowledge that these professionals have. On the other hand, the database environment in which the information is stored, and of course, where and how the data is stored, is something that most domain experts would have difficulties to explain, and although domain experts already have a deep under-standing of the variables and information within a dataset, is not always easy for them to know where to find (and how to access) such information. Also, data is usually spread across many tables within the database, and examining the datasets one by one, trying to find the right infor-mation to be used in the analysis, can be very time consuming and demotivating. Therefore, the first topic that should be highlighted during the Data Understanding stage is the understanding the data environment. Second, as domain experts are limited to time and also technical con-straints, it is not feasible to expect that any complex analysis or algorithms applications will be made during the investigation, hence, it is expected that they would only perform simple explor-atory data analysis which could be performed entirely during this phase.

Moving forward, as it should be clear now, preparing data can be very time consuming depending on the data quality level one wants to achieve. For some data mining methods, ensuring that only valid and clean data enters into the analysis is mandatory for a good outcome, as it is the case for classification and predictive DM methods for example. However, that does not mean that for the other types of DM activities data quality is less important, yet, by means of explora-tory data analysis and knowledge over the quality issues, problems can be considered and avoided during the analysis, and the quality improvements, if required, made on demand. The Data Preparation goal for domain experts, based on their technical skills, time constraints, and type of DM orientation to be pursued, should only focus in making the dataset simpler and smaller for further analysis. Based on all that, any proposed solution has to be straightforward, since, besides the time constraint aspect, people tend to get demotivated if stuck into something for too long with no much progress. Nevertheless, as it is hard to specify the exact activities that should be pursued in this phase (given that many activities are highly situational), two aspects can be highlighted, as ones that fit domain experts' needs (based on the difficulties stated by them during the interviews) and constraints, and at the same time, aligned with the phase's goal stated above: creating a unified view for the data, and constructing the dataset with the purpose of making any analytical task easier afterwards.

Based on has been said above, an adaptation for the CRISP-DM framework is proposed when focusing on domain experts. Thus, the three first phases from the model are suggested to be enough for them to pursue an analytical task without major help from data experts. The Business Understanding phase, should still be the starting point still, however, with the objective of only translating the research question or hypothesis that domain experts most of the times already have into an analytical project goal. Next, considering all that has been said above about the Da-ta Understanding and Data Preparation phases, the certainty that preparing the data is most of the times needed to the full comprehension of the data content and to perform a full data quality assessment, and the fact that it was suggested for domain experts to in fact pursue the whole da-ta analysis within the Data Understanding phase, two alterations are proposed in the original CRISP-DM model, as can be seen in Figure 1.

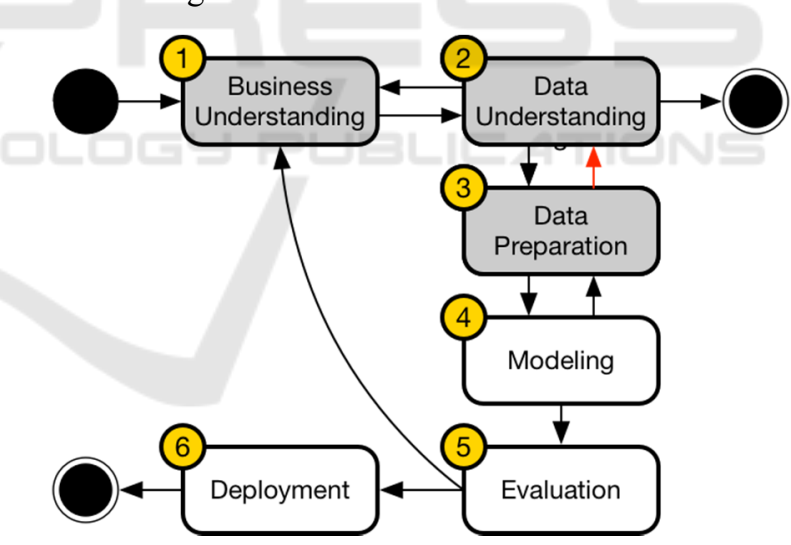

Figure 1: CRISP-DM for Domain Experts: Phases.

First, a two-way relationship was added between Data Understanding and the Data Preparation phases (arrow in read). That way, is possible to prepare and manipulate the data prior or during the exploratory analysis, as well as (if desired) to fully examine data quality problems within the data. That was an unexpected non-existent relationship in the original CRISP-DM, given the fact that even data analysts in order to fully understand the data, take advantage of some data preparation tasks in order to the explore it. Second, a new ending point was added after the Data Understanding phase. Thus, the process now has two 


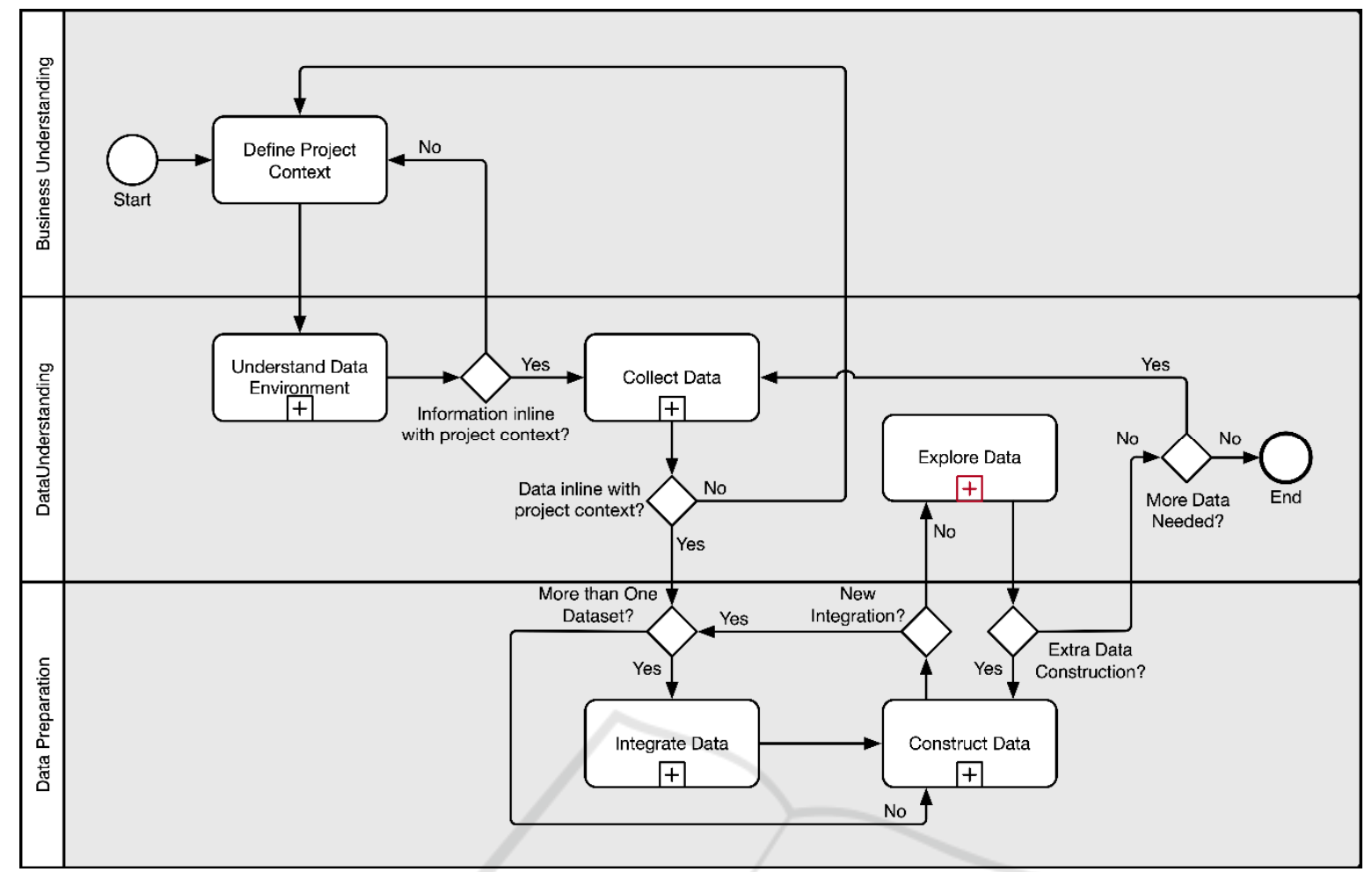

Figure 2: Knowledge Discovery Process for Self-Service Data Science.

ending points depending on the activity to be done, and the type of user who is conducting the analysis. The ending point after the Data Understanding phase would mean that domain experts would have concluded the exploratory data analysis, answered their research questions, and no further interactions are needed. Finally, the steps in grey are the ones suggested for domain experts.

\section{META-ALGORITHMIC MODEL}

The concept of MAM was inspired by the Method Engineering discipline, which is defined as a discipline to "design, construct and adapt methods, techniques and tools for the development of information systems" (Brinkkemper, 1996). In turn, MAM is given the meaning of the "engineering discipline where sequences of algorithm selection and configuration activities are specified deterministically for performing analytical tasks based on problem-specific data input characteristics and process preferences" (Spruit \& Jagesar, 2016). Thus, its main objective is to devise a step-by-step guideline, composed by method fragments, used to guide experts from the application domain (without deep technical expertise) in the understanding of some design science's artefact. These fragments were built using the Process-Deliverable Diagram (PDD) notation, which consists of two integrated diagrams that express both process-view and deliverable-view of an artefact construction. In this section the method fragments that are suggested to be followed by domain experts are illustrated bellow together with their brief description respectively. Figure 2 shows how each task from the MAM refers to the CRISP$\mathrm{DM}$ adaptation showed in the previous section.

\subsection{Understand Data Environment}

The first main activity is called 'Understand Data Environment' and it contains five sub-steps as depicted in Figure 3. It starts from the assumption that documents that describe in detail the database schema from a given business are updated and available for checking. Hence, first those documents have to be identified and retrieved, where explanations about the data tables within the database should exist. After that, one should focus on finding the information focusing in the data component in which the analysis should be based upon; verifying which data tables are available, their meanings, their purposes, and how they are arranged; identifying how data tables relate 
to each other, that means, which variables and attributes allow the establishment of a relationship between two tables; and finally understanding which variables are being stored within each table, and how to refer to them. No content is being analysed so far, only attributes and their meanings.

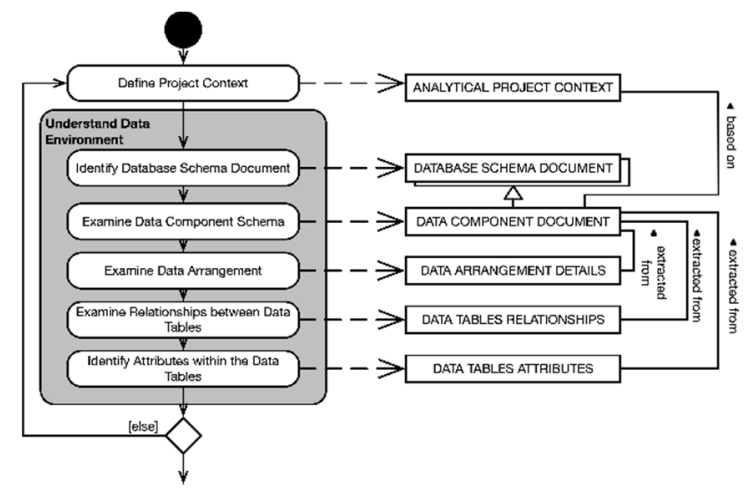

Figure 3: Understand Data Environment step.

\subsection{Collect Data}

After acquiring a reasonable knowledge over the data environment, the "Collect Data" activity has the purpose of loading the data files that are required for the analytical task and getting familiarized with them. Thus, this activity has three components, as shown in Figure 4 . The process starts by loading the data file into a tool of choice, such as loading a .CSV file into Excel or R. Next, is recommended to describe the data and then plot its features to start exploring the data and the relationship between its variables. It has the purpose of being a straightforward activity that aims to provide an overall picture of the data's content, such as some descriptive statistics, how the data is distributed, and some of its quality problems such as quantity of missing data related to a given variable.

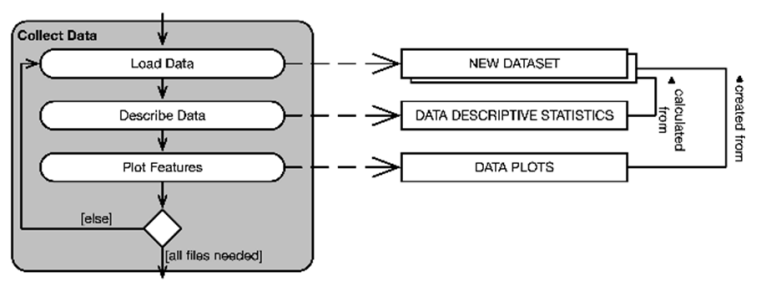

Figure 4: Collect Data step.

\subsection{Integrate Data}

Moving forward, after collecting and understanding the data, the acquired datasets should be prepared for the analysis accordingly to the user needs. Based on what has been explained in previous sections, the main objective of the Data Preparation phase for domain experts should be on creating a simplified and smaller dataset for an exploratory data analysis. Thus, considering the user group to whom this MAM is being created, the first main activity that are suggested is the 'Integrate Data' task, as shown in Figure 5.

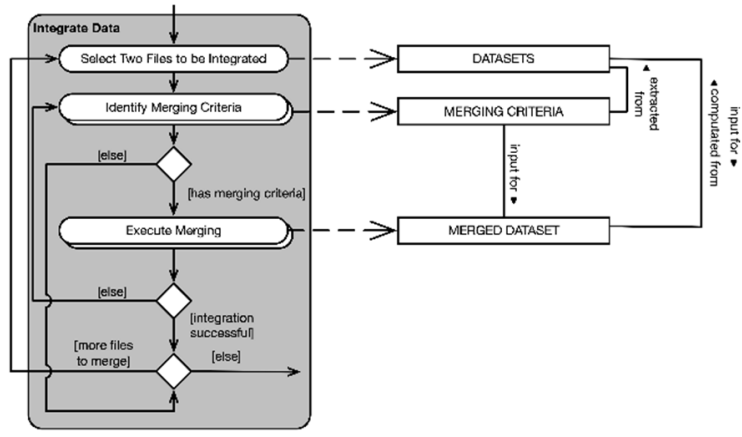

Figure 5: Integrate Data step.

Integrating datasets can be a tricky activity for those who do not have experience doing it, and it should be pursued of course, if more than one dataset has been collected/created. First, one has to know what can be integrated and what makes sense integrating. For domain experts, most of the information needed about this matter should have been acquired during the 'Understand Data Environment' activity, where the data tables and their relationship were examined. Thus, the 'Integrate Data' activity starts with the selection of the two convenient datasets to be integrated. After that, the merging criteria between those files have to be identified, where the correct and successful identification of such criteria is mandatory for a successful integration between the two datasets. Thus, the last activity, 'Execute Merging' should only be pursued if the merging criteria are indeed found.

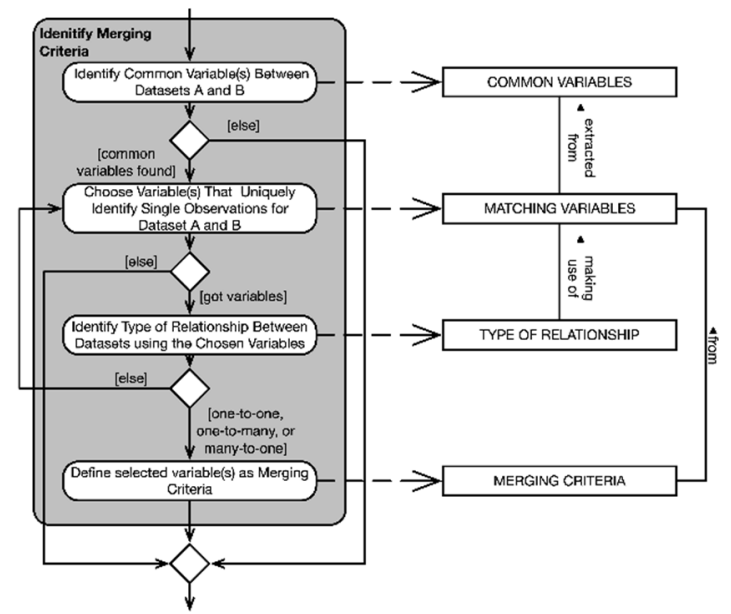

Figure 6: Identify Criteria substep within Integrate Data. 
Moreover, the merging criteria are usually defined by a primary and foreign key relationship between two data tables, that is, a common attribute that allows to identify matching records between two datasets. The method fragment shown in Figure 6 was designed to help domain experts on identifying those attributes. First, a user has to identify common variables (even if with different names) between both datasets, and then, the one(s) that uniquely identify single observations for each dataset, that is, the attribute(s) that permits to differentiate one record from another.

Furthermore, the next step is to identify the type of relationship (in terms of cardinality) between the two datasets considering the selected attributes as the merging criteria. Four distinct types of relationship cardinality exist: One-to-One (1:1), One-to-Many (1:n), Many-to-One (n:1), and Many-to-Many (n:n). Although in theory datasets could be merged despite the type of relationship, for the domain experts only the first three mentioned are suggested to be used, since the Many-to-Many could create very complex datasets, with several duplicate records, and even wrong information.

The last step for integrating the data is the actual execution of the merging task, as shown in Figure 7. It can be pursued in several ways, using different tools and notations. Moreover, the goal is not enforcing the user to choose one tool, and teach how to execute such task, but to provide the knowledge of what is needed in order integrate datasets. Thus, despite the means, the parameters which are required doing so are basically the same in any tool available. Thus, the only missing parameter is the merging type, which represents the definition of the content that should be returned after the conclusion of the merging task. Four merging types (the most commonly used and known) are suggested, they are: Inner Join, Left Join, Right Join, and Outer Join. Inner Join is probably the most commonly used merging type, and it returns all records from Dataset A which have a corresponding matching record in Dataset B. The Left Join type returns all record from Dataset A regardless if that record has a match or not in Dataset B, together with the matching records (if any) from Dataset B. Right Join, similarly to the Left Join, returns all record from Dataset B regardless if that record has a match or not in Dataset A, together with the matching records (if any) from Dataset A. Finally, the Outer Join merging type returns all records from both tables, matches and un-matches.

After choosing the right merging type for the given situation, all three main components for integrating two datasets were identified, what allows the merging task execution to be done, using the most convenient method and tool for the user. In $R$ for example, one could use the merge function and add the correct parameters based on the MAM above. The whole data integration process can be repeated if the merging was not successfully done (which could happen due to a bad judgment of the merging criteria) or if there are more files collected in previous steps to be merged.

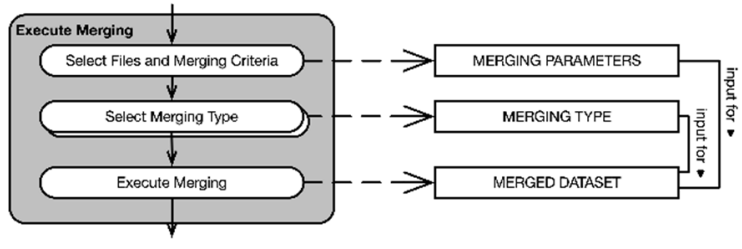

Figure 7: Execute Merging substep within Integrate Data.

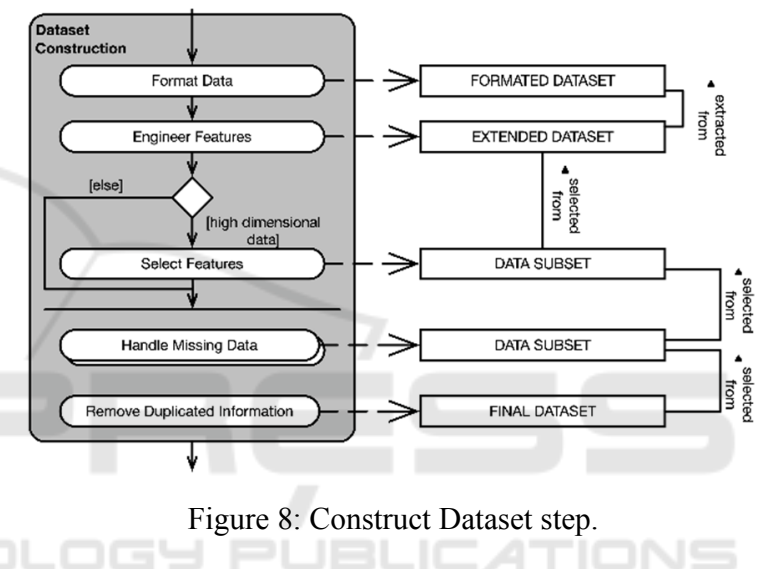

\subsection{Construct Dataset}

The last main activity within the Data Preparation phase is called "Construct Dataset". It comprises most of the tasks defined by the original CRISP-DM framework for the Data Preparation phase, such as data selection, formatting, construction, and data cleaning. This activity is suggested to be pursued with the dataset resulted from the 'Integrate Data' task or with the datasets initially collected. The activity is illustrated in detail in Figure 8.

It starts with the "Format Data" activity which is basically the same as the Data Formatting step from the CRISP-DM. Examples of tasks that can be done within this activity are: rearranging attributes, changing text from upper to lower case, etc., with the goal of formatting the variables without changing their meaning, building that way a better visualization (based on the user's interpretation) of the dataset to be analysed. The next proposed activity is called "Engineer Features", where new attributes can be constructed if needed based on the already existing attributes within a dataset. For example, if the dataset 
has the weight and height information from a given person, a new variable could be their body max index, calculated based on the existing variables.

Next, as proposed by Spruit \& Jagesar (2016), if the dataset is high dimensional, that is, if it has a high number of variables and records, a feature selection should be done, first to reduce the size of the dataset which will facilitate the analysis, and second, to remove variables and records that may not be relevant to the project goal and analytical task. Thus, the feature selection can be done both horizontally and vertically, where horizontally means applying feature selection techniques (like a simple filtering) to the attributes (columns) of a given dataset. On the other hand, vertically means applying those techniques upon the records (rows) from the dataset.

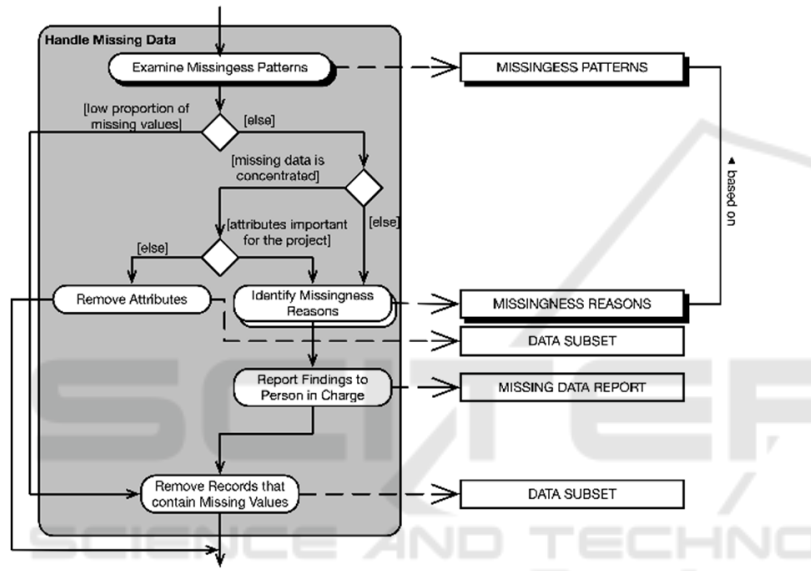

Figure 9: Handle Missing Data substep within Construct Dataset.

Finally, the last two activities are dedicated to handle the missing data and removing duplicate information, as shown in Figure 9. Thus, by cleaning the data building a simpler dataset, it should be easier to achieve and produce better results during the exploratory data analysis.

Additionally, missing data if not identified and considered during the analytical task, can heavily interfere in outcome by making the analysis biased due to the incomplete information. Thus, identifying and handling missing data is of high importance for any analytical activity. Moreover, when focusing in domain experts, is suggested that the user examine the missingness patterns of the given dataset. Two main aspects should be noticed when examining the patterns: the proportion of missing data when compared with the content of the dataset, and if it is

2 The Prototype Application can be accessed at: https://github.com/Dedding/R-Shiny-Prototype-Tool scattered throughout the many variables or concentrated in only few attributes. In the end, the main suggestion still is to remove all missing information, since even if the mechanisms of missingness (MCAR, MAR and MNAR) are well known, is not guaranteed that by using the existing methods and techniques such as deletion, single and multiple amputation approaches, and maximum likelihood estimation, to fix that specific issue will result in an optimal dataset (Baraldi \& Enders, 2010). Thus, what is suggested is to evaluate the missingness scenario, and to try removing the less number of records possible.

After constructing the dataset, the user has then the option of pursuing an exploratory data analysis with the new dataset, always being able to collect new data if needed, performing new integrations or constructing activities.

\section{EVALUATION AND RESULTS}

In order to evaluate the proposed guideline, first a structured walkthrough was performed, which is the process of explaining in detail every aspect of the artefact, with the purpose of ensure comprehension from the domain experts over the model, as well as guide them through why such activities exist, their expected outcomes, decisions that were made in order to create a given activity, what should be the benefits of using the guideline, and answer any questions they had (Rozanski \& Woods, 2005). Thus, it was mandatory to show why and how the proposed artefact would indeed be of value for the domain experts, meeting their needs, and making them understand the importance of using it. The next step of the evaluation procedure was to present a developed prototype tool ${ }^{2}$ to the participant, which although not being part of the final artefact and main deliverable of this study, it was developed with the objective of facilitating comprehension over the guideline, regarding its activities and their expected outcomes. Furthermore, with the purpose of exemplifying the model usage in the real-world, two use case scenarios were created, where an analytical project goal was defined in which domain experts had to go through the model by using real data extracted from the hospital's databases, with the aid of the prototype tool. After concluding both case studies, a questionnaire was applied where questions regarding the four topics: 
- Interpretability: accordingly to Bibal \& Frénay (2016), it can be explained by the following three connected subjects: understandability, accuracy, and efficiency. The first one means that a model is only interpretable when it can be understood. Accuracy refers to how accurate the model is to the data in hand since a model can be rather simple and easily understood without having any relationship with the data. Finally, efficiency, refers to the time and effort it takes to understand the model, however, this was not measured as it would not make sense for this study.

- Perceived Usefulness: refers to the degree to which the participant considers the artifact effective for structuring and preparing the data for an analytical project.

- Ease of Use: measures the degree to which the participant considers following the guideline free of effort

- Intention to Use: like its own name says, whether the participants intent to use the guideline for future analytical projects.

Finally, the data was analyzed, and the results are displayed below. The participants included in the validation process were five domain experts from the medical domain.

\subsection{Interpretability}

In terms of understandability, the ratings, as per the respondents, were considered very good, as they declared being able to fully comprehend the activities and outcomes from the guideline, and the importance of specific activities such as Understand Data Environment and Integrate Data, which were designed to facilitate not only the current step in the process, but its following activities. In terms of accuracy, it was clear that the participants felt confident on how real datasets from their domain fitted the MAM, and how the tasks and problems could be represented and assessed by following it. Therefore, the overall interpretability from the model, after conducting the evaluation, was considered high and fulfilled the expectations.

\subsection{Ease of Use}

To evaluate the overall ease of use of the MAM, three topics had to be considered: how much effort it took to understand the guideline, how much effort it took to follow it, and if the tool influenced positively (or not) in the evaluation of that matter. First, the effort to understand the model was not optimal nor high, it was rated to be between an average level of effort and almost effortless. As domain experts are not used to perform such activities, hence, it was expected for them to have some difficulties interpreting all activities and seeing the big picture immediately. Thus, that supported the choice of pursuing a structured walkthrough technique in the evaluation procedure. On the other hand, after the understanding of the MAM, the participants rated as almost effortless to follow its activities. In addition to that, most participants had good opinions about whether the tool helped on understanding the model and how easy was to use it. However, as it was limited to some small number of functionalities, the data preparation activity was limited to a pre-defined set of possibilities to be performed. Hence, that may have influenced negatively some ratings. Nevertheless, although a little bit of effort was needed to fully comprehend the model, it was possible to see a good evaluation of its ease of use.

\subsection{Perceived Usefulness}

Regarding the perceived usefulness, the majority of the respondents declared that they perceived the MAM to be indeed useful. Only one participant rated it as average. However, the level of usefulness of the guideline is directly influenced by the level of experience that one might have in the subject. Regarding this specific participant, as she had a little bit more experience on the subject, she did not need guidance for all activities depicted within the model. Moreover, she also stated that she missed some activities within the model to better handle missing data. However, as mentioned earlier, the model was built for an audience without prior experiences with data analytics and on preparing data, hence it had to be kept simple to what was feasible to the majority of this professionals. Therefore, considering the target audience and the problems that they face when trying to do KD, the overall perceived usefulness of the model fulfil the expectations.

\subsection{Intention to Use}

Last but not least, most domain experts declared that they indeed intent to use the model in future activities. In the same way to what happened on the perceived usefulness evaluation, only one participant rated her intention to use the MAM as average, which again relates to her level of experience on the matter and which tasks she intent to perform, as explained above. Therefore, as $80 \%$ of the respondents declared that they have the intention to use the model, it also achieved the expectation on this matter. 


\section{CONCLUSION}

Data analytics, as could be seen throughout this document, is a very promising and important field nowadays, as it is still growing and being adapted within many companies around the globe. The paper Power to the People! (Spruit \& Jagesar, 2016) represented a starting point for spreading the power of $\mathrm{KD}$, of technology, to people who are no experts in the area, who have other qualities that could indeed help on extracting information as good as (or sometimes better) data analysts or scientists. This study followed the same line of research, focusing in the applied data science area of study, and showed to be significant, as we could see it to be of value for domain experts to start exploring data in a simpler and structured way, as per the good results during the evaluation of the guideline.

Additionally, answering the research question presented earlier in this research, first, the original CRISP-DM was identified as being indifferent regarding the type of professional who is following it, when in reality the type of user who is conducting the analysis, in conjunction with the type of analytical project and data available, should determine how to pursue an activity, and which tasks to actually perform. Thus, an adaptation of the CRISP-DM was proposed, aligning the objectives of the framework with what is believed to be indeed important for domain experts (based on the interviews, data quality assessment, and literature review), where only the activities (as well as their inner tasks) that would add some value into the analysis, and at the same time, would be feasible considering all the mentioned constraints, were suggested to be followed by domain experts. Second, regarding the Data Preparation phase, one cannot prepare any data without first defining a project context and going through the Data Understanding phase. It was not possible to focus only in the Data Preparation task, without providing domain experts the means and the goals for preparing the data. Thus, to facilitate the Data Preparation phase the Business Understanding and Data Understanding phases had to be addressed and simplified as well. Third, as mentioned earlier in this study, Data Preparation is considered to be even more time consuming and complicated than DM itself. Defining how to pursue this activity, depends most of the times to the project at hand and information available. Thus, in order to facilitate it, the goals of this phase had to be limited to only making the dataset simpler and smaller, instead of fixing and cleaning all possible scenarios, given domain experts' time and technical constraints. Additionally, based on the difficulties mentioned by domain experts during the interviews and the quality of the data that they would be dealing with, some activities within the Data Preparation phase were highlighted, such as Data Integration and Data Construction, focusing on allowing those professionals to prepare the data, and at the same time, to not spend more time than required on this task. Therefore, Data Preparation for domain experts such as healthcare professionals should not have the purpose of creating a perfect dataset, but rather to create a simpler and smaller one for further exploration.

\section{REFERENCES}

Baraldi, A. N., \& Enders, C. K. (2010). An introduction to modern missing data analyses. Journal of School Psychology, 48(1), 5-37.

Bibal, A., \& Frénay, B. (2016). Interpretability of Machine Learning Models and Representations. ESANN European Symposium on Artificial Neural Networks, 2729.

Brinkkemper, S. (1996). Method engineering: Engineering of information systems development methods and tools. Information and Software Technology, 38(4), 275-280.

Cao, L. (2012). Actionable knowledge discovery and delivery. Wiley Interdisciplinary Reviews: Data Mining and Knowledge Discovery, 2, 149-163.

Chapman, P., Clinton, J., Kerber, R., Khabaza, T., Reinartz, T., Shearer, C., \& Wirth, R. (2000). Crisp-Dm 1.0. CRISP-DM Consortium.

Fayyad, U., Piatetsky-Shapiro, G., \& Smyth, P. (1996). From Data Mining to Knowledge Discovery in Databases. AI Magazine, 17(3), 37-54.

Francis, J., Johnston, M., Robertson, C., Glidewell, L., Entwistle, V., Eccles, M., \& Grimshaw, J. (2010). What is an adequate sample size? Operationalising data saturation for theory-based interview studies. Psychology and Health, 25(10), 1229-1245.

Linden, A., Vashisth, S., Sicular, S., Idoine, C., Krensky, P., \& Hare, J. (2017). Magic Quadrant for Data Science Platforms. G00301536. Gartner.

Luo, Q. (2008). Advancing Knowledge Discovery and Data Mining. First International Workshop on Knowledge Discovery and Data Mining (WKDD 2008), 7-9.

Raghupathi, W., \& Raghupathi, V. (2014). Big data analytics in healthcare: promise and potential. Health Information Science \& Systems, 2(1), 3.

Rozanski, N., \& Woods, E. (2005). Software Systems Architecture: Working with Stakeholders using Viewpoints and Perspectives. Addison-Wesley.

Spruit, M., \& Jagesar, R. (2016). Power to the People! - MetaAlgorithmic Modelling in Applied Data Science. Proc. of the 8th Int. Joint Conf. on Knowledge Discovery, Knowledge Engineering and Knowledge Management, $1,400-406$.

Tsai, C.-W., Lai, C.-F., Chao, H.-C., \& Vasilakos, A. V. (2015). Big data analytics: a survey. Journal of Big Data, 2(1), 21. 